Almeida, O.P. (2012). Approaches to decrease the prevalence of depression in later life. Current Opinion in Psychiatry, 25(6), 451-456.

Copyright: @ 2012 Lippincott Williams \& Wilkins, Inc.

This is pre-copy-editing, author-produced version of an article accepted for publication in Current Opinion in Psychiatry following peer review. The definitive published version (see citation above) is located on the journal home page of the publisher, Lippincott, Williams $\&$ Wilkins.

This version was made available in the UWA Research Repository on the $1^{\text {st }}$ of November 2013] in compliance with the publisher's policies on archiving in institutional repositories.

Use of the article is subject to copyright law. 


\section{APPROACHES TO DECREASE THE PREVALENCE OF DEPRESSION IN LATER LIFE}

Osvaldo P. Almeida

- Professor and Winthrop Chair of Geriatric Psychiatry, School of Psychiatry \& Clinical Neurosciences, University of Western Australia

- Director of Research, Western Australian Centre for Health \& Ageing, Centre for Medical Research, University of Western Australia

- Consultant Psychiatrist, Department of Psychiatry, Royal Perth Hospital

Prof. Osvaldo P. Almeida, WA Centre for Health \& Ageing (M573), University of Western Australia, 35 Stirling Highway, Crawley, Perth, WA 6009, Australia. Email: osvaldo.almeida@uwa.edu.au. T: 6189224 2855, F: 61892248009. 


\section{Purpose of the review}

Depression is a common and disabling disorder that affects people of all ages and $10 \%$ of those older than 65 years. Current strategies to decrease the personal and societal burden of depression in older age rely on the management of people with depression or of those at risk, but benefits have been limited and data remain scant.

\section{Recent findings}

Existing data suggest that decreasing the onset of a depressive disorder in people at risk is a reasonable way of reducing its prevalence in the community. Older adults with subthreshold symptoms of depression and those who suffered a stroke have been successfully targeted with various interventions. In addition, the use of collaborative care has improved the immediate and long-term outcome of patients with depression. Finally, the findings of epidemiological studies indicate that a more systematic approach to the management of risk factors might improve the outcome of patients and those at risk, but trial data are still lacking.

\section{Summary}

Current evidence suggests that it is possible to decrease the prevalence of depression in later life, but a more systematic approach to the assessment and management of older adults is necessary.

\section{Key-words}

Depression, elderly, prevention, treatment, risk factors. 


\section{INTRODUCTION}

Depression is a common mental disorder that affects people of all ages and about $10 \%$ of those older than 60 years living in the community $[1,2]$. Current estimates indicate that depression is the leading cause of years of life lost due to disability in both high and middle income countries [3], which may seem surprising given that effective treatment is widely available. This apparent contradiction can be partly explained by suboptimal access of patients with depression to services $[4,5]$ and to the limited efficacy of standard treatments [6-8]. Hence, there are 2 options to decrease the growing public health burden associated with depression worldwide: (1) prevent the development of depression in people at risk (i.e., reduce the incidence of depression); (2) improve access to services and the effectiveness of antidepressant treatments (i.e., decrease the prevalence of depression).

\section{CAN WE PREVENT DEPRESSION IN LATER LIFE?}

Ideally, effective preventive strategies should successfully target factors that are causally related to depression. The problem is that establishing causality in psychiatry is not a simple task, even though tentative guidelines have been available for nearly 5 decades [9]. Recent pragmatic approaches to the prevention of depression have focused on older people with symptoms of depression that do not reach threshold for the diagnosis of a depressive disorder [10], the socalled 'indicated prevention' [11]. A good illustration of such a study is the Preventive Intervention for Frail Elderly, the PIKO project. van't Veer-Tazelaar and colleagues [12] selected a sample of 170 adults aged 75 years or over who scored 16 or more on the Center for Epidemiologic Studies Depression Scale (CES-D) but did not fulfill DSM-IV criteria for a depressive or anxiety disorder at the time of assessment or during the preceding 12 months. The investigators implemented a stepped care intervention that consisted of 'watchful waiting' for the first 3 months, bibliotherapy (if subsequent $C E S-D \geq 16$ ), followed by problem solving therapy and, lastly, treatment with antidepressant medications. The relative risk of a depressive or anxiety disorder in the intervention compared with the usual care control group was 0.49 (95\% confidence interval, $95 \% \mathrm{Cl}=0.24-0.98)$ after 12 months. Four participants met criteria for a depressive disorder, 3 for an anxiety disorder, 
and 2 for mixed anxiety and depression in the intervention group compared with 10 cases of depressive disorder, 5 of anxiety and 5 mixed states in the usual care group. However, there was no statistically significant difference between the groups when the outcome of the study was limited to depression. In addition, dropout was higher in the intervention than in the usual care group $\left(24 / 86\right.$ vs $\left.8 / 84 ; x^{2}=3.68, p=0.002\right)$, suggesting the existence of problems with the acceptability of the intervention amongst participants.

Another example of 'indicated prevention' was carried out in Australia and involved 909 people aged 60 to 74 years [13]. Participants with a score of 16 or more on the Kessler Distress 10-scale (K10) were randomly assigned to treatment with folic acid (400 $\mu \mathrm{g} /$ day $)+$ vitamin B12 (100 $\mu \mathrm{g} /$ day $)$, physical activity, mental health literacy, or control activities (placebo tablets, nutrition promotion and pain management) in a crossed $2 \times 2 \times 2$ factorial design. By the end of the 24 months of treatment, there was no evidence that the use of B-vitamins or physical activity decreased the severity of depressive symptoms (measured with the Patient Health Questionnaire, PHQ-9), although a beneficial effect of literacy during the initial 6 weeks of the intervention was noticed, but not sustained. Of note, the intervention was delivered by post and via telephone contacts.

Other studies have targeted populations at high risk of depression, such as post stroke patients, using an approach commonly known as 'selective prevention'. Summarizing the findings of these studies has proven difficult because of the great heterogeneity of the populations under investigation, the interventions used, and the varying follow up times [14]. Nonetheless, some trials have found that the use of antidepressants or problem solving therapy reduces the risk of depression over a period of 12 months in post stroke patients [15], whilst others have found no benefit associated with active treatment [16]. A secondary analysis of data from a randomized placebo-controlled trial designed to reduce the risk of cardiovascular events found that post stroke patients treated with folate, B12 and B6 (mean age 63 years) had 50\% lower hazard of developing depression during the 7 years of treatment than patients treated with placebo, although the authors acknowledged that confounding and bias could have contributed to the results [17"]. 
A recent review of indicated or selective depression prevention trials across the lifespan reported data on 33 trials, most of which used cognitive behavioural or problem solving therapies as the active intervention [18]. The authors found an overall benefit of the interventions (about $25 \%$ risk reduction compared with control interventions), although the non-independence of some studies was not taken into account in the analyses (i.e., trials with more than one intervention group were treated as separate studies). In addition, there was some evidence of publication bias, which creates uncertainty regarding the validity of the summary findings.

Other approaches used with the aim of reducing the prevalence of depression in the community have focused on improving the identification of people with depression [19-23] and on increasing the efficacy and effectiveness of existing management strategies [8, 24]. Screening and casefinding are the approaches most frequently employed to identify people with depression in populations at risk, particularly in general practice settings. However, a meta-analysis of 16 randomized trials found that the systematic use of screening instruments or case-finding procedures increased the recognition of depression by $27 \%$, but had no obvious effect on the adoption of treatments or the clinical outcome of patients [25]. Similarly, the evidence in support of the use of educational interventions targeting medical practitioners to reduce the prevalence of depression remains inconclusive.

\section{WHAT ARE THE BEST OPTIONS TO TREAT DEPRESSION IN LATER LIFE?}

The successful management of older adults with depression is important not only to decrease personal suffering and direct and indirect health burden, but also to decrease the number of people with depression living in the community. In other words, the more people we treat successfully, the less people with depression will exist (see figure). At an individual level, we should aim to use therapies that have great efficacy and tolerability, so as to increase the odds of remission and compliance with treatment. Pharmacological treatment with antidepressants in later life has proven efficacy compared with placebo [26, $\left.{ }^{\bullet}\right]$, particularly when used by patients with moderate to 
severe illness [23"]. Overall, 40 to $70 \%$ of patients experience clinically significant improvement when they use these medications [26], and there are no compelling data suggesting that a particular medication or group of medications has greater efficacy than others. In addition, and contrary to widespread belief, there is limited hard evidence that older adults tolerate serotonin reuptake inhibitors (SSRIs) better than treatment with trycyclic antidepressants [28, 29].

There are few randomised trials of psychological interventions for older people with depression, and empirical support for their systematic use in clinical practice is weak [30]. There is some evidence that these interventions may mitigate the severity of depressive symptoms (particularly interpersonal psychotherapy) [31], but concerns remain regarding the generalizability of findings and whether psychological interventions afford additional benefit to patients when compared to antidepressant treatment alone [32, 33].

Psychosocial interventions have also been used to treat depressive symptoms in later life with or without antidepressants. Older adults with mild to moderate symptoms of depression seem to benefit from various forms of treatment such as physical activity, skill training, social activities and group support, but again data are limited and existing findings are difficult to generalise and implement in clinical practice [34].

Electroconvulsive therapy $(E C T)$ use is commonly restricted to cases of severe or psychotic depression that are unresponsive to other forms of treatment [35]. However, randomised controlled trials of ECT in later life depression are all but non-existent [36], creating uncertainty about how best to justify its use in clinical practice.

Taken together, currently available evidence indicates that antidepressant interventions can reduce the prevalence of depression in later life. However, existing trial data are mostly limited to the healthy young old, and there is a paucity of information about how best to manage those aged 75 years or over or who present with multiple comorbidities. Similarly, further research is required 
to guide the management of the substantial proportion of older people who fail to respond well to a standard antidepressant medication or to psychotherapy.

\section{IS IT POSSIBLE TO AVOID RELAPSE AND RECURRENCE OF DEPRESSION?}

Depression is often the expression of a recurrent disorder, so that avoiding the relapse or recurrence of symptoms is an important approach to reduce the prevalence of depression in older age. It is, therefore, disappointing that only 8 small to medium size randomised trials have investigated this issue systematically over follow up periods ranging from 6 to 24 months [37"]. They found that a lower proportion of older adults who continued to use antidepressant medication after successful treatment of the index depressive episode experienced recurrence of symptoms compared with those assigned placebo ( $25 \%$ vs $52 \%)$. The number of people needed to treat to avoid a relapse or recurrence was $3.6(95 \% \mathrm{Cl}=2.8-4.8)$, which suggests that treatment maintenance should be encouraged beyond the initial successful management of the acute episode. Psychological interventions, such as interpersonal psychotherapy, may be as effective as antidepressant medications in preventing the relapse or recurrence of symptoms [33], although systematic data on this topic remain sparse.

\section{APPROACHES DESIGNED TO OPTIMISE THE MANAGEMENT OF DEPRESSION}

Ten years ago Unützer and colleagues reported the results of a collaborative care trial designed to improve the health outcomes of older people with depression, the IMPACT study [38]. They recruited over 1800 older adults with major depression or dysthymia who were randomly assigned to usual care or an intervention that included supervised case management, education, support of antidepressant use and brief problem solving therapy. After 12 months, $45 \%$ of patients in the intervention group had improved compared with only $19 \%$ of those receiving usual care (odds ratio, $\mathrm{OR}=3.4,95 \% \mathrm{Cl}=2.7-4.4)$. Gains were sustained over a follow up period of 2 years [39]. Other trials that used collaborative care models to manage older adults with depression treated in primary care settings have reported similarly encouraging results [12, 40, 41"'], and there is now evidence that people with chronic illnesses can also benefit from treatment [42-" $]$. Notwithstanding the fact that 
the gains associated with this intervention are modest and the costs high $[43,44]$, the introduction of collaborative care management represents a concrete step forward in the effort to reduce the prevalence of depression in older age.

More recently, Almeida and colleagues reported data from a large cross-sectional study ( $n>20,000$ people aged 60 years or over) that investigated the contribution of multiple factors on the risk of depression in later life [45 $\left.^{\mathbf{D}}\right]$. They created a risk matrix that included demographic, developmental, lifestyle, socioeconomic and clinical risk factors for depression, and suggested that the cumulative stratification of exposures could assign participants to various probabilistic levels of depression that ranged from less than $3 \%$ to slightly more than $80 \%$. The novelty of the proposed approach is that such a matrix could potentially be used in clinical practice to guide interventions aiming to reduce the prevalence of depression in older age. For example, a 76 year-old obese gentleman who is a smoker, lost his father at the age of years and received only two years of primary school education has a $20-30 \%$ probability of experiencing clinically significant depressive symptoms in later life. In this case, if this person were to give up smoking, become more physically active and reduce his $\mathrm{BMI}$ to 28 , the probability of depression could potentially drop by $20-25 \%$ if the risk factors are causally related to depression. The availability of such information offers clear incentives to the patient and the clinician to consider and address relevant factors that are amenable to change and that are likely to produce the greatest reduction in the risk of depression. Despite the inherent attraction of such an approach, confirmatory longitudinal and trial data are required to test its usefulness in clinical practice.

\section{FIGURE}

\section{CONCLUSION}

The figure shows various pathways associated with depression that could potentially be targeted to reduce the prevalence of depression in the community. Remission of depression amongst cases and prevention amongst those free of symptoms are the ultimate goal. Until now, our approach has 
been mostly limited to the management of those with depression and, more recently, people with subthreshold illness. Notwithstanding concrete progress on that front, data on how best to treat and prevent depression in later life remains scant and limited to the effects of antidepressants and some forms of psychotherapy. Future studies may benefit from integrating information about known risk factors for depression into their interventions [45", 46] with the aim of determining how this might change the outcome of patients and of those at risk.

- Trial evidence shows that collaborative care and stepped-care programs decrease the prevalence of depression in later life by enhancing treatment response and reducing the incidence of depressive and anxiety disorders in older people with subthreshold depressive symptoms.

- Antidepressant medications, some forms of psychotherapy and use of certain vitamins hold potential to reduce the incidence of clinically significant depressive symptoms in specific populations at high risk for depression, such as post-stroke patients, but data remain scant and difficult to generalise.

- The management of older adults at risk of depression should include the systematic assessment and management of relevant risk factors, and not be limited to the use of antidepressant medications and psychotherapy. 


\section{Acknowledgement}

The drafting of this manuscript was partly supported by a project grant from the National Health and Medical Research Council of Australia (NHMRC 572594). The sponsors had no role in the design and conduct of the study; collection, management, analysis, and interpretation of the data; or preparation, review, or approval of this manuscript.

Conflict of interest declaration

The author declared he has no conflict of interest. 


\section{REFERENCES AND RECOMMENDED READING}

- $\quad$ of special interest

- of outstanding interest

[1] Beekman AT, Copeland JR, Prince MJ. Review of community prevalence of depression in later life. Br J Psychiatry 1999; 174:307-311.

[2] Prince M, Patel V, Saxena S et al. No health without mental health. Lancet 2007; 370:859877.

[3] Mathers C, Boerma T, Ma Fat D. The global burden of disease: 2004 update. Geneva: World Health Organization; 2008.

[4] Akincigil A, Olfson $M$, Siegel $M$ et al. Racial and ethnic disparities in depression care in community-dwelling elderly in the United States. Am J Public Health 2012; 102:319-328.

[5] Andrews G, Sanderson K, Slade T, Issakidis C. Why does the burden of disease persist? Relating the burden of anxiety and depression to effectiveness of treatment. Bull World Health Organ 2000; 78:446-454.

[6] Cipriani A, Furukawa TA, Salanti G et al. Comparative efficacy and acceptability of 12 newgeneration antidepressants: a multiple-treatments meta-analysis. Lancet 2009; 373:746-758.

[7] Cuijpers P, van Straten A, Warmerdam L, Andersson G. Psychotherapy versus the combination of psychotherapy and pharmacotherapy in the treatment of depression: a metaanalysis. Depress Anxiety 2009; 26:279-288.

[8] Kirsch I, Deacon BJ, Huedo-Medina TB et al. Initial severity and antidepressant benefits: a meta-analysis of data submitted to the Food and Drug Administration. PLoS Med 2008; $5: e 45$.

[9] Hill AB. The environment and disease: association or causation? Proc R Soc Med 1965; 58:295-300.

[10] Lyness JM, Yu Q, Tang W et al. Risks for depression onset in primary care elderly patients: potential targets for preventive interventions. Am J Psychiatry 2009; 166:1375-1383.

[11] Sriwattanakomen R, Ford AF, Thomas SB et al. Preventing depression in later life: translation from concept to experimental design and implementation. Am J Geriatr Psychiatry 2008; 16:460-468.

[12] van't Veer-Tazelaar PJ, van Marwijk HW, van Oppen $P$ et al. Stepped-care prevention of anxiety and depression in late life: a randomized controlled trial. Arch Gen Psychiatry 2009; 66:297-304.

[13] Walker JG, Mackinnon AJ, Batterham $P$ et al. Mental health literacy, folic acid and vitamin B12, and physical activity for the prevention of depression in older adults: randomised controlled trial. Br J Psychiatry 2010; 197:45-54.

[14] Hackett ML, Anderson CS, House AO, Halteh C. Interventions for Preventing Depression After Stroke. Stroke 2009.

[15] Robinson RG, Jorge RE, Moser DJ et al. Escitalopram and problem-solving therapy for prevention of poststroke depression: a randomized controlled trial. JAMA 2008; 299:23912400. 
[16] Almeida OP, Waterreus A, Hankey GJ. Preventing depression after stroke: Results from a randomized placebo-controlled trial. J Clin Psychiatry 2006; 67:1104-1109.

[17] Almeida OP, Marsh $\mathrm{K}$, Alfonso $\mathrm{H}$ et al. B-vitamins reduce the long-term risk of depression after stroke: The VITATOPS-DEP trial. Ann Neurol 2010; 68:503-510.

- This is subgroup analysis of a larger randomised trial designed to investigate if the use of vitamins B6, B9 and B12 could reduce the recurrence of cardiovascular events after a stroke. The investigators found that the incidence of depression was lower amongst those treated with vitamin than with placebo.

[18] Munoz RF, Cuijpers P, Smit F et al. Prevention of major depression. Annu Rev Clin Psychol 2010; 6:181-212.

[19] Kessler D, Lloyd K, Lewis G, Gray DP. Cross sectional study of symptom attribution and recognition of depression and anxiety in primary care. BMJ 1999; 318:436-439.

[20] Pfaff JJ, Almeida OP. A cross-sectional analysis of factors that influence the detection of depression in older primary care patients. Australian and New Zealand Journal of Psychiatry 2005; 39:262-265.

[21] Arroll B, Elley CR, Fishman T et al. Antidepressants versus placebo for depression in primary care. Cochrane Database Syst Rev 2009:CD007954.

[22] Rush AJ, Trivedi MH, Wisniewski SR et al. Acute and longer-term outcomes in depressed outpatients requiring one or several treatment steps: a STAR*D report. Am J Psychiatry 2006; 163:1905-1917.

[23] Fournier JC, DeRubeis RJ, Hollon SD et al. Antidepressant drug effects and depression severity: a patient-level meta-analysis. JAMA 2010; 303:47-53.

- This comprehensive meta-analysis confirmed the efficacy of antidepressants compared with placebo, particularly when used by patients with severe illness.

[24] Corey-Lisle PK, Nash R, Stang P, Swindle R. Response, partial response, and nonresponse in primary care treatment of depression. Arch Intern Med 2004; 164:1197-1204.

[25] Gilbody S, Sheldon T, House A. Screening and case-finding instruments for depression: a meta-analysis. CMAJ 2008; 178:997-1003.

[26] Nelson JC, Delucchi K, Schneider LS. Efficacy of second generation antidepressants in latelife depression: a meta-analysis of the evidence. Am J Geriatr Psychiatry 2008; 16:558-567.

[27] Tedeschini E, Levkovitz $\mathrm{Y}$, lovieno $\mathrm{N}$ et al. Efficacy of antidepressants for late-life depression: a meta-analysis and meta-regression of placebo-controlled randomized trials. $\mathrm{J}$ Clin Psychiatry 2011; 72:1660-1668.

- This paper summarises useful efficacy data from placebo-controlled trials of antidepressants in later life.

[28] Wilson K, Mottram P. A comparison of side effects of selective serotonin reuptake inhibitors and tricyclic antidepressants in older depressed patients: a meta-analysis. Int J Geriatr Psychiatry 2004; 19:754-762.

[29] Mittmann N, Herrmann N, Einarson TR et al. The efficacy, safety and tolerability of antidepressants in late life depression: a meta-analysis. J Affect Disord 1997; 46:191-217. 
[30] Wilson KC, Mottram PG, Vassilas CA. Psychotherapeutic treatments for older depressed people. Cochrane Database Syst Rev 2008:CD004853.

[31] Cuijpers P, Geraedts AS, van Oppen P et al. Interpersonal psychotherapy for depression: a meta-analysis. Am J Psychiatry 2011; 168:581-592.

[32] Reynolds CF, 3rd, Dew MA, Martire LM et al. Treating depression to remission in older adults: a controlled evaluation of combined escitalopram with interpersonal psychotherapy versus escitalopram with depression care management. Int J Geriatr Psychiatry 2010; 25:1134-1141.

[33] Reynolds CF, 3rd, Frank E, Perel JM et al. Nortriptyline and interpersonal psychotherapy as maintenance therapies for recurrent major depression: a randomized controlled trial in patients older than 59 years. JAMA 1999; 281:39-45.

[34] Forsman AK, Schierenbeck I, Wahlbeck K. Psychosocial interventions for the prevention of depression in older adults: systematic review and meta-analysis. J Aging Health 2011; 23:387-416.

[35] Kho $\mathrm{KH}$, van Vreeswijk MF, Simpson S, Zwinderman AH. A meta-analysis of electroconvulsive therapy efficacy in depression. J ECT 2003; 19:139-147.

[36] Flint AJ, Gagnon N. Effective use of electroconvulsive therapy in late-life depression. Can J Psychiatry 2002; 47:734-741.

[37] Kok RM, Heeren TJ, Nolen WA. Continuing treatment of depression in the elderly: a systematic review and meta-analysis of double-blinded randomized controlled trials with antidepressants. Am J Geriatr Psychiatry 2011; 19:249-255.

- Clinical impression suggests that continuation of antidepressant treatment is an effective way of decreasing relapse and recurrence of symptoms. This meta-analysis of 8 randomised trials confirms that this is indeed the case, but also shows that at leat $1 / 4$ patients will relapse within 6-24 months despite ongoing treatment.

[38] Unutzer J, Katon W, Callahan CM et al. Collaborative care management of late-life depression in the primary care setting: a randomized controlled trial. JAMA 2002; 288:28362845.

[39] Hunkeler EM, Katon W, Tang $L$ et al. Long term outcomes from the IMPACT randomised trial for depressed elderly patients in primary care. BMJ 2006; 332:259-263.

[40] Bruce ML, Ten Have TR, Reynolds CF, 3rd et al. Reducing suicidal ideation and depressive symptoms in depressed older primary care patients: a randomized controlled trial. Journal of the American Medical Association 2004; 291:1081-1091.

[41] van't Veer-Tazelaar PJ, van Marwijk HW, van Oppen $P$ et al. Prevention of late-life anxiety and depression has sustained effects over 24 months: a pragmatic randomized trial. Am J Geriatr Psychiatry 2011; 19:230-239.

- This paper reports data from a seminal Dutch trial (reference 12) showing that a steppedcare program decreased the one-year incidence of depressive or anxiety disorders in older adults with subthreshold symptoms of depression (CES-D score $\geq 16$ ).

[42] Katon WJ, Lin EH, Von Korff $\mathrm{M}$ et al. Collaborative care for patients with depression and chronic illnesses. N Engl J Med 2010; 363:2611-2620. 
- Collaborative care has changed the way the management of depression is approached in primary care. This study shows that collaborative care works even in the presence of concomitant chronic illnesses, which is relevant for a large proportion of older adults living in the community.

[43] Schoenbaum M, Unutzer J, Sherbourne $C$ et al. Cost-effectiveness of practice-initiated quality improvement for depression: results of a randomized controlled trial. JAMA 2001; 286:1325-1330.

[44] Gilbody S, Bower P, Whitty P. Costs and consequences of enhanced primary care for depression: systematic review of randomised economic evaluations. Br J Psychiatry 2006; 189:297-308.

[45] Almeida OP, Alfonso $\mathrm{H}$, Pirkis $\mathrm{J}$ et al. A practical approach to assess depression risk and to guide risk reduction strategies in later life. Int Psychogeriatr 2011; 23:280-291.

- This observational study shows that it is possible to predict the probability of depression in later life by analysing associated risk factors. The authors built a probabilistic matrix that followed the same principles used to calculate the risk of cardiovascular events, and suggested that this information could be used to guide management and the introduction of consumer-tailored preventive strategies.

[46] Smit F, Smits N, Schoevers $\mathrm{R}$ et al. An epidemiological approach to depression prevention in old age. Am J Geriatr Psychiatry 2008; 16:444-453. 


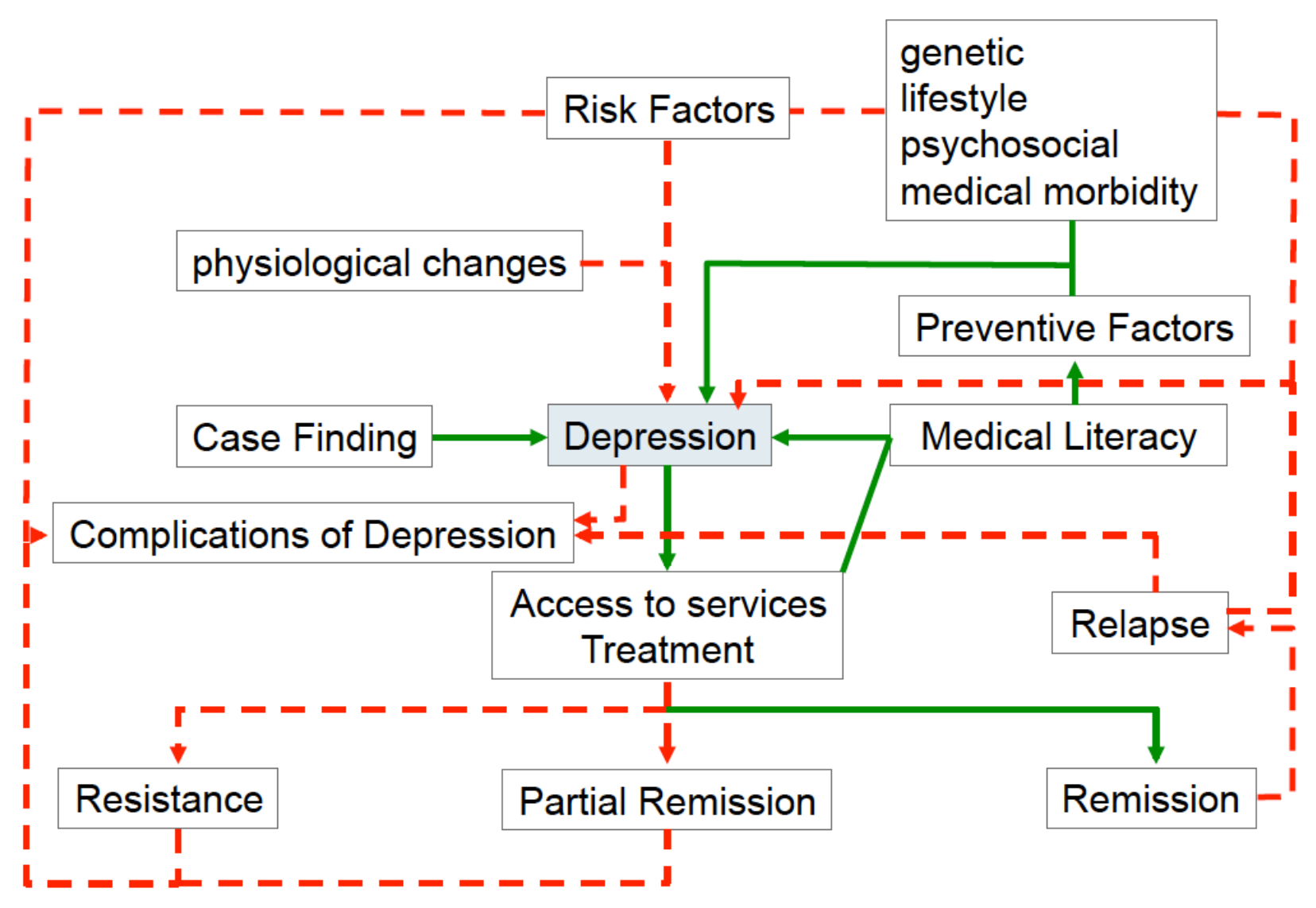

Figure 1. The figure depicts pathways associated with depression that may be amenable to intervention. Full arrows indicate that an increase in that pathway has the potential to decrease the prevalence of depression, whereas dashed lines indicate that a decrease in the relevant pathway is required to decrease the prevalence of depression. 